Colorectal cancer (CRC) is one of the most common malignancies worldwide. In recent years novel therapies targeted at EGFR receptor have been developed. However, this treatment can only be beneficial if no mutation in specific loci of KRAS/NRAS and $B R A F$ genes is found in tumour specimen. Therefore, clinically useful pathological diagnosis of CRC in the era of personalised medicine is a multistep procedure, requiring good cooperation between the clinician/ surgeon, pathomorphologist, and molecular biologist. Herein we propose the guidelines of colorectal cancer operating material proceedings for clinicians and pathomorphologists, which determines the correct pathomorphological diagnosis, and we discuss the colorectal cancer molecular biology issues useful in the selection of individual molecular targeted therapy. We discuss and stress the importance of each diagnostic phase: from tumour resection and sample collection at preanalytical stage, through proper pathological preparation, evaluation and selection of material for molecular testing, to molecular analysis and finally preparation of a pathological molecular report.

Key words: colorectal cancer, KRAS, NRAS, BRAF, diagnostic, mCRC.

Contemp Oncol (Pozn) 2018; 22 (2): 75-85 DOI: https://doi.org/10.5114/wo.2018.77047

\section{Quality and practical aspects of pathological and molecular diagnostics in metastatic colorectal cancer (mCRC)}

\section{Andrzej Tysarowski ${ }^{1,2}$, Anna Nasierowska-Guttmejer ${ }^{3,4}$}

${ }^{1}$ Department of Pathology and Laboratory Medicine, Maria Skłodowska-Curie Memorial Cancer Centre and Institute of Oncology, Warsaw, Poland

${ }^{2}$ Department of Molecular and Translational Oncology, Maria Skłodowska-Curie

Memorial Cancer Centre and Institute of Oncology, Warsaw, Poland

${ }^{3}$ Pathology Department, The Jan Kochanowski University in Kielce, Poland

${ }^{4}$ Pathology Department, Central Clinical Hospital of the MSWiA in Warsaw, Poland

\section{Introduction}

In 2013, colorectal cancer (CRC, including colon and rectal cancers) was the second most common female cancer (7173 cases, accounting for 9.2\%), and a third of all tumours diagnosed among males (8726 cases, 11.2\%) in Poland. Furthermore, CRC contributed to the demise of 5851 men and 4711 women, which made it the second and the third cause of cancer-related deaths in 2013, respectively. According to global data, in 2012, colorectal cancer was diagnosed in a total of 1.4 million patients and was the third most common cancer worldwide [1].

The methods of colorectal cancer treatment are determined by several factors, of which tumour location (colon vs. rectum) and staging according to TNM, are the most important. In recent years treatment targeted at the selective inhibition of tumour cell growth using small molecule inhibitors and monoclonal antibodies has been introduced. This method of therapy is largely based on the knowledge of tumour biology. Hence, the challenge for modern molecular diagnostics is to enable precise selection of patients, who can benefit from different treatment strategies.

Targeted therapies in colorectal cancer are primarily directed at blocking the epidermal growth factor receptor (EGFR receptor), which is involved in signalling pathways controlling cell proliferation, angiogenesis, apoptosis, and metastasis. Overexpression of the receptor, observed in $45-80 \%$ of patients with CRC, is associated with worse prognosis: a higher cancer grade, lymph node involvement, and a higher frequency of metastasis to the liver and to lungs, bones, etc. [2-4].

In colon cancer cells, especially in the deepest layers of the tumour, besides EGFR overexpression, higher concentration of receptor-activating ligands is also observed. Importantly, during activation of the receptor, signalling crosstalk and cross-phosphorylation may occur. This allows for the mutual activation of neighbouring receptors or interactions between different signalling pathways. During neoplastic transformation, the self-activation processes, in which the cell itself produces ligands for receptors on its own surface, might be possible as well [4-6].

Currently, the most efficient blocking of EGFR-dependent pathways is achieved using two monoclonal antibodies: cetuximab and panitumumab, which bind the extracellular domain of the EGFR receptor, preventing its dimerisation and self-activation. As a result, signalling transduction, synthesis, and secretion of proangiogenic factors are reduced, the cell cycle is arrested, and pro-apoptotic processes are strengthened. These agents also 
induce an antibody-dependent cell mediated cytotoxicity (ADCC) reaction [7].

However, targeted inhibition of the EGFR is ineffective when further components of signal pathway are damaged. In normal cells, the EGFR signal is passed through one of the three main signalling pathways: the RAS/RAF/MAPK/ ERG pathway, the PI3K/AKT pathway, and the STAT pathway. Therefore, exclusion of KRAS, NRAS and BRAF mutations in the tumour is one of the main inclusion criteria for targeted therapy using anti-EGFR antibodies.

Monoclonal antibody therapies targeting epidermal growth factor receptor (anti-EGFR therapy) are currently used in Poland as a first-line treatment for patients with advanced CRC. Cetuximab and panitumumab may be used in monotherapy or in combination with chemotherapy.

Importantly, anti-EGFR therapy can only be included after confirmation of CRC diagnosis by pathomorphological examination and exclusion of mutations in selected regions of KRAS, NRAS, and BRAF genes.

Mutation status of those genes is carried out using molecular techniques that enable detection of somatic changes in FFPE (formalin-fixed and paraffin-embedded) tumour samples. Because the percentage of cells containing mutations in the examined material can be very low, high-sensitivity detection methods of molecular diagnostics can only be used. Similarly, proper preparation and precise pathomorphological characteristics of the colorectal cancer histopathological samples for molecular diagnostics are critical.

The role of pathomorphologist in contemporary oncological diagnostics is to recognise cancer, determine its histological type, degree of maturity, and stage of advancement (PTNM), and to evaluate prognostic and predictive factors. Thus, pathomorphological diagnosis is crucial with respect to the decision of treatment strategy in an individual patient. Consequently, the full clinically useful pathological diagnosis of CRC in the era of personalised therapy depends on good cooperation between the clinician/surgeon, pathomorphologist, and molecular biologist.

Hence, the aim of this paper is to:

- propose the guidelines of colorectal cancer operating material proceedings for clinicians and pathomorphologists, which determines the correct pathomorphological diagnosis,

- discuss the colorectal cancer molecular biology issues useful in the selection of individual molecular targeted therapy.

\section{The pathological and molecular analysis of the tumour tissue}

The European Society of Pathology and Royal College of Pathologists has recently elaborated the guideline for laboratories performing molecular pathology for cancer patients [8]. In the new situation in oncology when personalised medicine requires evaluation of predictor factors using molecular tests, evidence-based recommendations should, in particular, be followed by clinicians/surgeons, pathologists, and molecular biologists. All diagnostic procedures, including DNA and RNA extraction, can and should be standardised and verified on a regular basis.

The diagnostic process of cancer specimens includes:

- the preanalytical stage,

- the diagnostic stage performed by the pathologists,

- the molecular analysis conducted by the molecular biologists,

- the final diagnosis prepared as a pathological molecular report.

\section{The preanalytical stage}

In the first step of the pre-analytic stage the tumour samples are taken from patients by the endoscopist or surgeon, are sent to the pathological laboratories, and are preserved and fixed.

The clinician who send the material for pathomorphological and/or molecular examination is obliged to correctly mark the container with the material transferred for examination and to deliver all the necessary information about the patient and the specimen. Complete clinical information is crucial for the pathological diagnosis.

A referral for a pathomorphological examination should contain, at least:

- personal data of the patient: name, surname, date of birth, PESEL or PID number marked with the traditional method or barcode,

- clinical diagnosis,

- anatomical location and type of surgical procedure with the marked surgical margins,

- in selected cases, a request for additional immunohistochemical or molecular tests for prediction factors assessment.

The pathologists receive different tissue material from the metastatic colorectal cancer cases: small biopsy from the primary tumour or from metastasis to the liver in inoperable cases or primary tumour specimens after surgery. The small biopsies are fixed rapidly in neutral buffered formalin (4\% formaldehyde) by endoscopists. Concerning the large specimen size after surgery, sending the fresh material to the laboratories is the most convenient. Because the tumour tissue requires controlled fixation, proceeding in the pathology laboratory is the optimal method. If the pathology laboratory is located in the hospital, non-fixed postoperative material should be sent there immediately. If the estimated transport time to the pathology laboratory is more than an hour, a vacuum is a preferable method for preservation of the fresh specimens. When the transport is expected to be longer, the surgeons should preserve and fix the specimen by themselves immediately after surgery in the operating theatre. If surgical material is sent to another remote location, the fixing conditions and time, together with transport procedure, must be observed by clinicians.

The surgeons should be familiar with the methods of preservation and fixation of large intestine tumour specimens. The preservation of the postoperative material includes cutting the large intestine along the long axis and unfastening it on an expanded polystyrene, wooden, paraffin, or cork board. The prepared specimen is fixed in 
$10 \%$ buffered formalin (4\% formaldehyde aqueous solution buffered to $\mathrm{pH}$ 7.2). Formalin is a commercially available fixative fluid, which is chemically a $40 \%$ solution of formaldehyde in water.

Formalin buffering should be obligatory because the formaldehyde sold commercially is often contaminated with various chemical substances and is acidic. This may adversely affect its fixing properties, hamper diagnostic processes, and result in the degradation of genetic material (DNA, RNA). In the case of large surgical specimens, a major problem for molecular pathology is a cold ischaemia occurring between removal of a tissue and its fixation, which alters levels of gene expression (at the RNA and protein level).

The specimen should be fixed in formalin for 48 hours or, preferably, for 72 hours. Longer and better fixation of the material allows removal of thinner sections, enabling better investigation of the extent of cancer infiltration. Proper fixation of the material should be achieved when 10 fixer volumes per one volume of the tissue material are provided. Formalin penetrates the tissue at a rate of $1 \mathrm{~mm} / \mathrm{h}$ to a depth of up to $5 \mathrm{~mm}$, thus material cutting ensures proper access to tissue penetration. Control of fixation time and temperature is recommended for molecular analysis. It should be emphasised that autolytic changes of the tumour occur if the uncut large intestine is inserted into the vessel, containing formalin volume less than required, which results in the inability to obtain the correct results of molecular tests. Poor fixation of the postoperative material is the first cause of the erroneous results of molecular analysis. The material destroyed at this stage is unrecoverable and it is impossible to correct the pre-analytic stage errors.

In the next step of the pre-analytic stage, the following procedures are performed:

a) macroscopic examination and collection the representative specimens by a pathomorphologist or doctor during specialisation with the participation of a laboratory technician/diagnostician,

b) placement of collected samples in the cassettes and signing them with the test number (entering bar codes in the LIS is suggested) by a laboratory technician/laboratory diagnostician manually or automatically with a barcode,

c) carrying out processing of the material in a tissue processor (reagents and handling times need to be checked),

d) paraffin embedding, slicing, handwriting, or automatic bar code signing and staining by a laboratory technician/diagnostician; it is necessary to control the appropriate paraffin temperature.

It should be emphasised that the tissue material should be processed through subsequent stages (points b, c, and d) in the pathology laboratory ensuring automation of the whole process. Equipment ensuring control of its conditions determines the appropriate quality of tissue material useful both for microscopic assessment and molecular testing. This is the important and obligatory condition affecting the quality of the obtained results. Hand-processing of tissue material should not be allowed during the preparation of histopathological preparations (Figs. 1 and 2).

\section{The diagnostic stage}

The final pathological diagnosis in colorectal carcinoma cases should be useful for clinicians in making decisions about treatment of patients. Molecular report in the era of personalised medicine should be integrated with the histopathological report. Pathologists use the synoptic reporting form based on a structured checklist. The checklist format guarantees the completeness of reporting for surgical pathology cases and production of the standardised clinical documentation. Pathologists can provide complete reports based on the College of American Pathologists (CAP) Cancer Protocols and electronic Cancer Checklists. The evolution from narrative reporting to pathology reporting using a configurable templates structured synoptic report
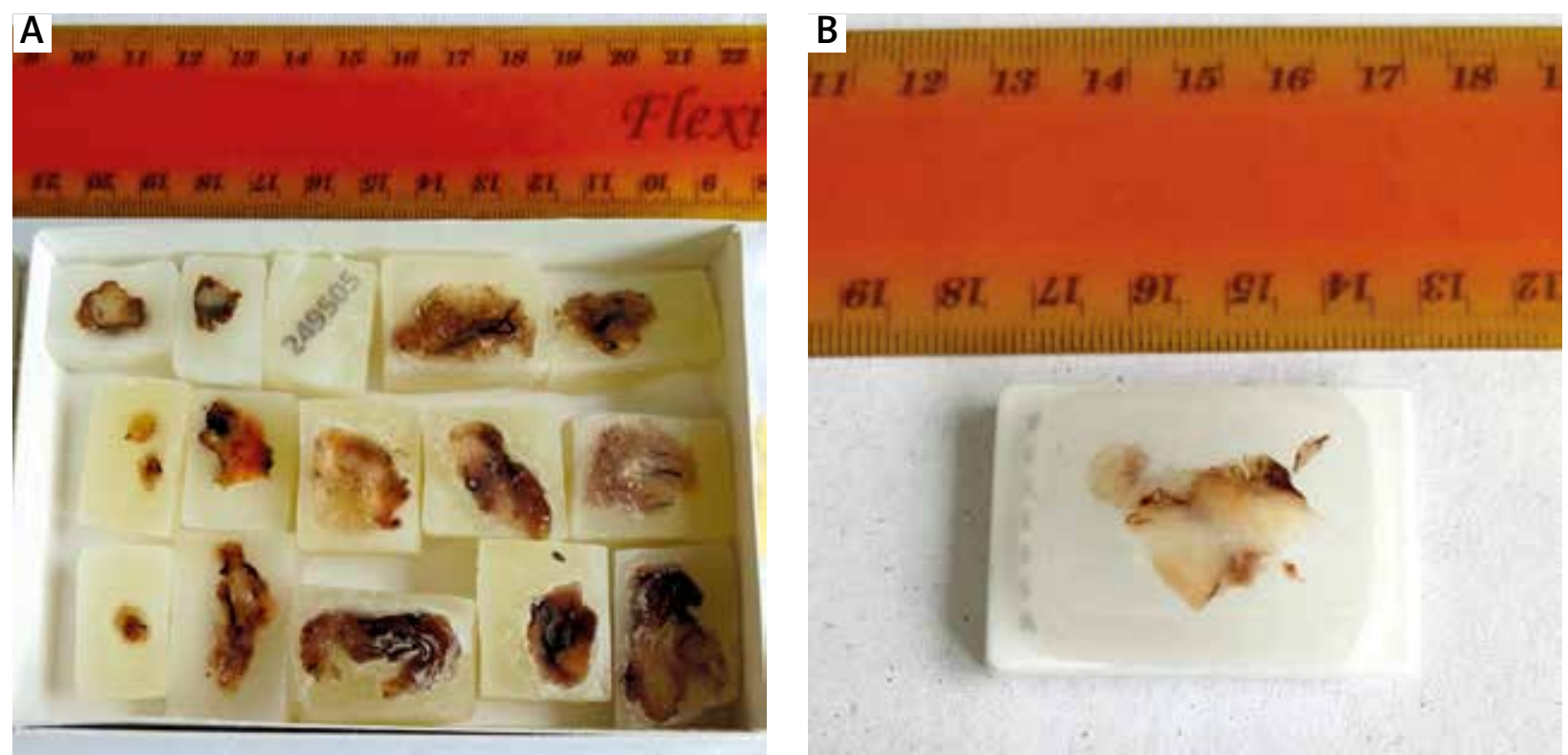

Fig. 1. A) The material prepared and processed by hand. B) The material placed in the cassette and carried out automatically in the processor 


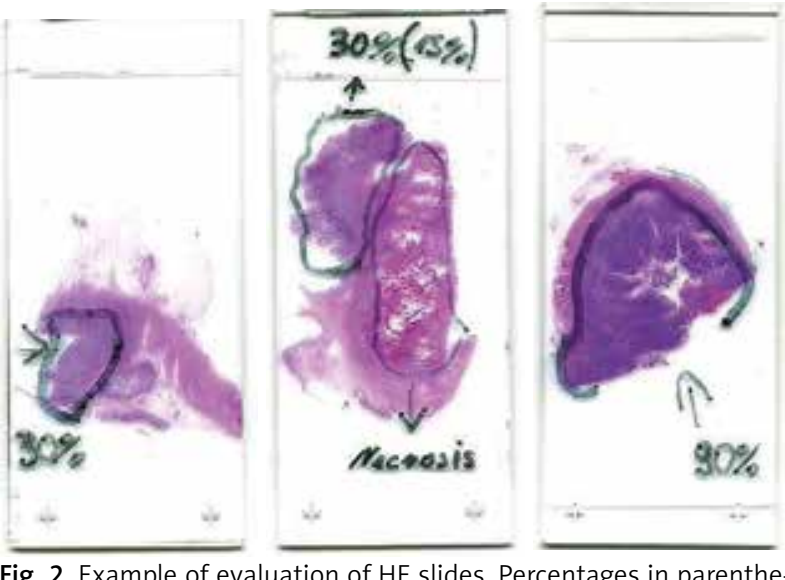

Fig. 2. Example of evaluation of HE slides. Percentages in parenthe ses indicate the evaluated percentage of tumour cells in the neoplasm areas marked by the pathologist

reduce the risk of lacking important, clinically useful information. A systemic review showed that synoptic reporting improved the completeness of pathology diagnosis and quality of pathology evaluation for solid tumours [9]. Besides traditional parameters for colorectal diagnosis, such as tumour type, grade, invasion depth (PT), and nodal status $(\mathrm{pN})$, other important clinical features are reported, e.g. resection margin, type of local spread (vascular, lymphovascular, perineural invasion), tumour deposits, and degree of tumour regression after preoperative treatment. Synoptic reports also include information about the mean number of lymph nodes examined. The quality of pathology reports is presented by the number of lymph nodes assessment (minimum 12 lymph nodes) and the proportion of the lymph nodes with carcinomatous metastasis to lymph nodes without metastasis.
Molecular pathological reports join the histopathological macro- and microscopic parameters with prognostic and predictive biomarkers. The colorectal pathological reports include molecular factors evaluated using immunohistochemical and molecular methods. The mutation status of KRAS, NRAS, and BRAF genes and microsatellite instability (MSI) are important biomarkers in the selection the patients for individual therapy.

The Eighth Edition of the TNM American Joint Committee on Cancer (AJCC) conformed the staging proposed by the TNM Committee of the International Union Against Cancer (UICC) [10, 11], which transformed the traditional version of the diagnostic reports into a more useful form for individualised therapy purposes. The main idea of the new version of TNM system is the term "prognostic stage groups" analysed separately for the tumour of the different anatomic site [12]. The pathological staging in colorectal cancers is based on the microscopic examination of the resected specimen (PTNM). The parameters defined for category $T$ (tumour), N (lymph nodes status), and M (distant metastasis) are required for stage grouping.

Additional factors recommended for clinical care are evaluated as tumour deposits (TDs). In the Seventh Edition of TNM TDs are classified as category pN1c. TD is defined as a nodule of regular or irregular contour of cancer cells situated in pericolic/perirectal fat tissue or in adjacent mesentery without lymph node metastasis. This microscopic parameter changes the stage of the tumour. This microscopic parameter changes the stage of the tumour and is classified as pN1c category. The factors that are important to consider in making a decision about treatment in colorectal cancer cases constitute another group of prognostic factors according to the Eighth Edition of the TNM AJCC/UICC. These parameters are clinically significant

Table 1. The factors important to consider in making a decision about treatment according to the Eighth Edition of the TNM AJCC/UICC [8]

Factor important to consider in making decisions about treatment

Serum carcinoembryonic antigen (CEA) levels in patients after surgery

Tumour regression score in rectal carcinoma after pre-operative treatment

Circumferential resection margin (CRM)

Lymphovascular invasion (LVI)

Perineural invasion (PNI)

Microsatellite instability (MSI)

KRAS and NRAS mutation status

BRAF mutation

\section{Comment}

CEA level may be measured in blood, plasma, or serum by an enzyme-linked immunosorbent assay (ELISA). It is recommended for monitoring the treatment. CEA levels may be used as a response marker for treatment of IV stage disease

The pathological response to preoperative radiotherapy (rectal cancer), chemoradiation (rectal cancer) or chemotherapy (colon and rectal cancer) is an important prognostic parameter assessed by the pathologists according to score system

CRM is measured in millimetres between the deepest margin of tumour tissue and the nonserosalised area of colorectum

In colorectal specimens the invasion of small and large vessels is associated with lymph node metastasis. The extramural venous invasion is an independent adverse prognostic parameter and risk factor for liver metastasis

The invasion of nerves and perineural spaces by the tumour cells correlate with a poor prognosis

MSI is a prognostic factor and predictive for lack of response to 5-fluorouracil (5-FU) chemotherapy. The MSI is a key to diagnose the hereditary nonpolyposis colorectal carcinoma (HNPCC) or Lynch syndrome. The sporadic colorectal cancer with MSI has epigenetic promoter methylation analysis of the MLH1. BRAF mutation and MSI in sporadic colon carcinoma predicts worse prognosis in stage III and IV colon cancer

The status of both genes is a predictive factor and correlates with lack of response to anti-EGFR therapy

BRAF p.Val600Glu mutation in colorectal carcinoma blocks the effect of anti-EGFR therapy and predicts progression in stage IV colorectal carcinoma 
when the physician and patient make a decision concerning treatment. Finally, the anatomical site of the tumour, histological type, grade, and pTNM should be considered together with these additional factors. Table 1 presents the factors to consider in making a decision about treatment according to the Eighth Edition of the TNM AJCC/UICC.

An additional part of the pathological report is dedicated to immunohistochemical and molecular analysis:

- immunohistochemical testing for mismatch repair (MMR) proteins (MLH1, MSH2, MSH6, and PMS2), molecular analysis of MMR genes using mononucleotide panel (BAT-25, BAT-26, NR-21, NR-24, and MONO-27), or other and MLH1 promoter methylation analysis,

- KRAS, NRAS, and BRAF mutational analysis.

\section{The molecular analysis}

\section{Preparation of material for diagnostic testing}

In the case of metastatic colorectal cancer, metastasis tumour (e.g. to the liver or lymph nodes) should be tested preferentially. However, if unavailable, the primary tumour tissue may be used.

In each case one of the most serious problems associated with the determination of somatic mutations in cancer tissue is its heterogeneity. The FFPE tumour samples commonly contain a significant percentage of normal cells, tumour microenvironment cells, such as activated fibroblasts, activated macrophages, lymphocyte and endothelial cells of the blood, and lymph vessels.

This issue is not a major concern in the aspect of histopathological diagnosis, but it limits significantly the possibility of isolating separated cancer cells for molecular examination, which can further lead to an erroneous test result. If the number of normal cells is much higher than the number of tumour cells, the signal from mutant DNA is "dimmed" by wild type DNA from normal cells, leading to a false negative result. In order to avoid this, the analysed sample should contain not less than $50 \%$ of neoplastic fixation to obtain a reliable result. Use of samples containing smaller proportion of tumour cells is permitted, although in such cases highly sensitive analytical methods, such as quantitative polymerase chain reaction (qPCR), should be used. However, according to recommendations, material for molecular purposes should contain tumour cells (almost) exclusively [13].

Therefore, a key element in the preparation of the sample for molecular analysis is the verification of the material by a pathologist, who: 1 . selects a slide containing the highest possible percentage of cancer cells in relation to the normal ones and 2. marks the area of tumour cells on the slide (Fig. 2).

In order to obtain a high percentage of cancer cells, manual separation of normal and cancerous tissue (macrodissection) on a slide is an option. The FFPE block can be also molten, so that the entire tumour tissue fragment can be macrodissected and subsequently re-embed in paraffin. Alternatively, microdissection can be performed using automated microdissectors. Such devices, usually coupled with scanners and microscopes, cut-out previously selected fragments of tissue sample from the slide and transfer it to the test tube. Laser micro-dissectors are even able to extract single cancer cells.

The number of sections should depend on the size of the cancerous tissue. In the case of large fragments (diameter of the section about $1 \mathrm{~cm}$ ), sections (of thick not exceeding 4-5 $\mu \mathrm{m}$ ) from 2-3 slides would be sufficient, while a minimum of five slides of small sections (with a diameter of 2-3 $\mathrm{mm}$ ) is required.

The thickness of the sections usually ranges from 4 to $10 \mu \mathrm{m}$. Thin sections allow faster isolation of genetic material, while thicker ones facilitate manual transfer to test tubes. Importantly, the pathologist is not able to evaluate the cells in the deeper layers of thicker sections and verify the presence of the tumour cells there. Furthermore, all tumour material in a block can be unintentionally used and will no longer be available for any future analyses.

It is also crucial to underline that the HE preparation for the assessment of neoplasm percentage must be made just before the preparators for molecular analysis; otherwise, the tumour content on the HE slide and slide(s) for molecular test may be dramatically different, which may negatively affect molecular test results.

\section{Nucleic acids extraction, quantification, and quality assessment}

Nucleic acid extraction from paraffin-embedded tissue should be efficient and enable purification from inhibitors of enzymatic reactions.

Currently, dedicated commercial kits seem to be the best choice. There are several sets available, both manual and compatible with automatic isolators. Extraction systems are usually based on columns containing solid phase binding DNA, or they use the technology of magnetic microspheres that capture DNA molecules and can be easily separated from the solution using magnetic stand.

The quantity and quality of isolated genetic material need to be evaluated prior to the analysis.

For this purpose, fluorimetric systems measuring the amount of DNA-binding substance should be used instead of spectrophotometers. The isolated form FFPE often contain many compounds that may interfere with the spectrophotometric absorbance measurements. This is of particular importance when the analysis is based on qPCR technology or next-generation sequencing, which require very precise amounts of nucleic acids.

Moreover, quantitative and quality assessment of DNA based on quantitative ( $q P C R$ ) reaction might be another option because it also enables assessment of the degree of DNA degradation and hence may indicate the necessity to resign from further analysis (if DNA is heavily degraded) and to repeat the isolation process from another slide.

Turnaround time in the case of molecular diagnostics depends on the mutation detection method. Real-time PCRbased methods are generally rapid because they demand one-step analysis. Sequencing methods, both classical (Sanger) and especially next generation (NGS), require much more time due to the multistep processes. While choosing the method of analysis, the following aspects should be a matter of careful consideration: availability of equipment 
and technical support, limitations of method optimisation, sensitivity and specificity of the method, time necessary to complete analysis, and unambiguity of the results.

\section{Final stage}

The complete diagnosis, that includes a pathological report and results of molecular tests for predictors, finishes the diagnostic process of cancer.

\section{Molecular predictors of response to targeted therapies in metastatic colorectal cancer}

Proteins involved the response to therapies targeted at $\mathrm{mCRC}$

RAS proteins belong to the family of $G$ proteins, which have an ability to bind and hydrolyse guanosine triphosphate (GTP). Binding the ligand by the EGFR receptor leads to the activation of the RAS family proteins, which are active when bound to GTP. Activated RAS conducts the sig nal further to the effector proteins, which include:

- family of RAF serine-threonine kinases: c-RAF, ARAF, and BRAF, which control cell proliferation and differentiation,

- 3-phosphatidylinositol kinase (phosphoinositide 3-kinase - PI3K), responsible for apoptosis regulation and cell survival,

- RALGDS, RGL, and RGL2 and TIAM1 proteins that activate RAL and RAC proteins; the RAL protein is responsible for the intracellular vesical transport, while the RAC protein is involved in the cytoskeleton modelling,

- C $\varepsilon$ phospholipase (PLC $\varepsilon$ ), involved in calcium-dependent signal regulation control.

RAS protein is inactivated through interaction with GAP proteins (GTP-ase-activating proteins), e.g. neurofibrin 1 (NF1) or p120. NF1 binds to the active RAS and activates its internal GTPase activity. As a result, the GTP hydrolysis process is a thousand-fold accelerated. Conversion of the RAS-GTP to RAS-GDP (inactive form) results in the inhibition of signal transduction.

The RAS subfamily consists of HRAS, NRAS, KRASA, and KRASB proteins, which are encoded by three distinct genes: HRAS, NRAS, and KRAS (encoding two isoforms: KRASA and KRASB - Fig. 3). Mutations in NRAS and KRAS genes have prognostic significance in the context of CRC therapy. At the protein level, mutation in these genes result in the abolition of the internal GTPase activity, making the protein insensitive to GAPs. Consequently, mutated
RAS proteins remain active and their accumulation leads to constant cell stimulation. Therefore, such mutations are referred to as activating [14-20].

The majority of activating mutations in NRAS and KRAS genes occur in codons 12 and 13, which accounts for $70 \%$, and $20 \%$ of all mutations identified in colon cancer, respectively. Mutations affecting codons 59, 61, 117, 146, and others are rarer (Table 2). RAS mutations are monoallelic changes occurring at the early stages of carcinogenesis (early and mid-advanced adenomas). However, in around $4 \%$ of cases mutations in codon 12 and 13 in tumour cells are identified simultaneously [21-24].

BRAF is another protein that has been indicated to play an important role in determining the response to targeted therapy in CRC. Activation of the BRAF protein, following phosphorylation by RAS kinase, leads to activation of another signalling protein - MEK kinase - and subsequently ERK kinase. ERK regulates gene expression and cytoskeleton rearrangement and is involved in several cellular processes: metabolism, proliferation, differentiation, aging, and apoptosis. In the inactive form, the conformation of BRAF protein's kinase domain prevents the binding of ATP (and the activation of MEK kinase). Structurally, this conformation is preserved by trapping the regulatory part of the enzyme, the activation segment, through hydrophobic interactions with the P loop. Indeed, the majority of activating mutations occur in loci involved in interaction of $\mathrm{P}$ loop and activation segment, which is critical to maintain the inactive form. As a result of mutation, the inactive form loses its stability in favour of the activated form $[28,29]$.

Mutations in BRAF occur in $5-22 \%$ of colorectal carcinomas. More than 40 different BRAF mutations in 24 different codons have been identified so far. In CRC, the most frequent (90\%) of them is the substitution of valine at position 600 with glutamate, which localises in the kinase domain. In in vitro kinase activity studies, the p.Val600Glu mutant is over 500 times more active than the normal form [28, 29].

Mutations in BRAF occur in the early stages of neoplastic transformation and are not capable of initiating the neoplastic process per se. However, for patients with colorectal cancer treated with cetuximab and panitumumab, the p.Val600Glu mutation is a negative predictor. Patients who have normal RAS genes and the mutant form of BRAF do not respond to treatment with anti-EGFR antibodies, and have shortened PFS and OS as compared to patients with wild type BRAF [30].

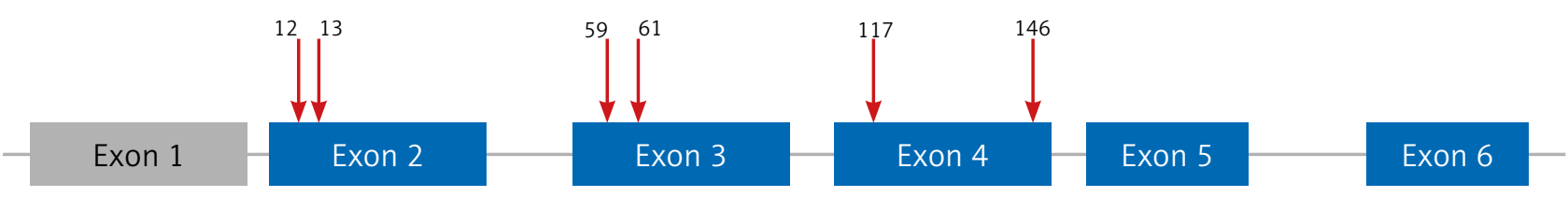

Fig. 3. The KRAS gene is located on the shorter arm of chromosome 12 (12p1 locus). It consists of six exons. The first exon, (grey) is not translated. Coding exons (blue) are numbered: 2, 3, 4, 5, 6. Red arrows indicate schematic position of codons (numbers) that are tested. Historically uncoding exon 1 was referred to as 0 ; hence, rarely in literature data the naming of the exons is shifted. Due to alternative splicing, two isoforms of KRAS are produced. The isoforms differ at their 3' ends: one contain exon 5 and the other exon 6 , historically named 4A and 4B, respectively. Hence, protein isoforms are named: KRAS 4A (189 amino acids) or KRAS 4B (188 amino acids). The KRAS 4B, which accounts for $90-95 \%$ of all KRAS produced in cells, has antiapoptotic functions and stimulates cell migration. On the contrary, KRAS $4 A$ has pro-apoptotic properties [25-27] 
Although literature data indicate that mutations in $B R A F$ and KRAS are mutually exclusive, there are sporadic reports of CRC tumours with coexisting KRAS and BRAF mutations [28-30]. Moreover, according to the recent American Society for Clinical Pathology, the College of American Pathologists, the Association for Molecular Pathology, and the American Society of Clinical Oncology guidelines, routine assessment of the BRAF status is only recommended for prognostic stratifications and in patients with deficient MMR tumours [31].

Nevertheless, there is a great deal of evidence that $B R A F$ mutations may negatively affect anti-EGFR therapy. Therefore, local guidelines regarding the spectrum of molecular testing prior to anti-EGFR therapy may differ. For example, in Poland exclusion of KRAS, NRAS, and BRAF mutations is mandatory for anti-EGFR therapy consideration [32, 33].

Molecular diagnostic of these genes is often performed in separated tests, one after another: KRAS, NRAS, and BRAF, according to mutation detection rate. If mutation is found in KRAS, analysis of (an)other gene(s) is not performed (as patient is already disqualified from anti-EGFR therapy).

Novel findings indicate, however, that in some CRC tumours other BRAF mutations are detected, which lead to silencing of BRAF kinase activity. Such patients may respond to EGFR therapy. Importantly, if they also have an activating mutation in KRAS/NRAS genes, they may benefit from another type of targeted therapy - using MEK inhibitors. Thus, one can expect that within the next years BRAF testing may become compulsory regardless of KRAS/ NRAS status [34].

\section{Modern methods of mutation detection}

Rapid development of molecular biology has enabled elaboration of many methods for DNA mutation detection (Table 3). The majority of them are based on PCR amplification of selected DNA fragments. The available methods, although widely used, differ in both the technical approach and sensitivity (defined as the minimal percentage of mutant cells that can be detected).

The simplest methods, usually qualitative ones, allow us only to determine if mutation(s) is/are present or not. More advanced techniques are based on modification of PCR reaction, require specialist equipment, and enable accurate determination of the mutation type. One of those techniques is direct sequencing, using Sanger method, which currently is called "a gold standard for mutation detection". Furthermore, the other technique - pyrosequencing - enables determination of the amount of mutant allele in relation to the normal tissue. Modern, expensive techniques based on real-time PCR technology allow analysis of a large number of samples in a short time, while very sensitive and precise tests based on next-generation sequencing technology (NGS) additionally allow the simultaneous detection of many other molecular markers.

There are also CE-IVD certified kits available, which can be used to comply with the European Union directives. It should be remembered, however, that diagnostic CE-IVD tests are valid only when performed on CE-IVD equipment.
Table 2. Mutations in the KRAS/NRAS/BRAF genes based on the COSMIC database

\begin{tabular}{|c|c|c|c|c|}
\hline Gene & Exon & Codon & $\begin{array}{c}\text { Mutation name } \\
\text { (protein level) }\end{array}$ & $\begin{array}{l}\text { Mutation name } \\
\text { (nucleotide level) }\end{array}$ \\
\hline \multirow[t]{26}{*}{ KRAS } & \multirow{12}{*}{2} & \multirow{6}{*}{12} & p.Gly12Asp & c. $35 \mathrm{G}>\mathrm{A}$ \\
\hline & & & p.Gly12Val & c. $35 \mathrm{G}>\mathrm{T}$ \\
\hline & & & p.Gly12Cys & c. $34 \mathrm{G}>\mathrm{T}$ \\
\hline & & & p.Gly12Arg & c. $34 \mathrm{G}>\mathrm{C}$ \\
\hline & & & p.Gly12Ser & c. $34 \mathrm{G}>\mathrm{A}$ \\
\hline & & & p.Gly12Ala & c. $35 G>C$ \\
\hline & & \multirow{6}{*}{13} & p.Gly13Asp & c. $38 \mathrm{G}>\mathrm{A}$ \\
\hline & & & p.Gly13Cys & c. $37 \mathrm{G}>\mathrm{T}$ \\
\hline & & & p.Gly13Arg & c. $37 G>C$ \\
\hline & & & p.Gly13Ser & c. $37 \mathrm{G}>\mathrm{A}$ \\
\hline & & & p.Gly13Ala & c. $38 \mathrm{G}>\mathrm{C}$ \\
\hline & & & p.Gly13Val & c. $38 \mathrm{G}>\mathrm{T}$ \\
\hline & \multirow{9}{*}{3} & \multirow{3}{*}{59} & p.Ala59Thr & c. $175 \mathrm{G}>\mathrm{A}$ \\
\hline & & & p.Ala59Glu & c. $176 \mathrm{C}>\mathrm{A}$ \\
\hline & & & p.Ala59Gly & c. $176 C>G$ \\
\hline & & \multirow{6}{*}{61} & p.Gln61Lys & c. $181 C>A$ \\
\hline & & & p.Gln61Lys & c. $180 \_181 \mathrm{TC}>\mathrm{AA}$ \\
\hline & & & p.Gln61Leu & c. $182 \mathrm{~A}>\mathrm{T}$ \\
\hline & & & p.Gln61Arg & c. $182 A>G$ \\
\hline & & & p.Gln61His & c. $183 A>C$ \\
\hline & & & p.Gln61His & c. $183 \mathrm{~A}>\mathrm{T}$ \\
\hline & \multirow{5}{*}{4} & \multirow{2}{*}{117} & p.Lys117Asn & c. $351 \mathrm{~A}>\mathrm{C}$ \\
\hline & & & p.Lys117Asn & c. $351 \mathrm{~A}>\mathrm{T}$ \\
\hline & & \multirow{3}{*}{146} & p.Ala146Thr & c. $436 G>A$ \\
\hline & & & p.Ala146Pro & c. $436 G>C$ \\
\hline & & & p.Ala146Val & c. $437 \mathrm{C}>\mathrm{T}$ \\
\hline \multirow[t]{18}{*}{ NRAS } & \multirow{8}{*}{2} & \multirow{5}{*}{12} & p.Gly12Cys & c. $34 \mathrm{G}>\mathrm{T}$ \\
\hline & & & p.Gly12Ser & c. $34 \mathrm{G}>\mathrm{A}$ \\
\hline & & & p.Gly12Ala & c. $35 G>C$ \\
\hline & & & p.Gly12Asp & c. $35 \mathrm{G}>\mathrm{A}$ \\
\hline & & & p.Gly12Val & c. $35 \mathrm{G}>\mathrm{T}$ \\
\hline & & \multirow{3}{*}{13} & p.Gly13Arg & c. $37 \mathrm{G}>\mathrm{C}$ \\
\hline & & & p.Gly13Val & c. $38 \mathrm{G}>\mathrm{T}$ \\
\hline & & & p.Gly13Asp & c. $38 \mathrm{G}>\mathrm{A}$ \\
\hline & \multirow{6}{*}{3} & 59 & p.Ala59Thr & c. $175 \mathrm{G}>\mathrm{A}$ \\
\hline & & \multirow{5}{*}{61} & p.Gln61Lys & c. $181 C>A$ \\
\hline & & & p.Gln61Leu & c. $182 \mathrm{~A}>\mathrm{T}$ \\
\hline & & & p.Gln61Arg & c. $182 A>G$ \\
\hline & & & p.Gln61His & c. $183 \mathrm{~A}>\mathrm{T}$ \\
\hline & & & p.Gln61His & c. $183 A>C$ \\
\hline & \multirow{4}{*}{4} & \multirow{2}{*}{117} & p.Lys117Asn & c. $351 G>C$ \\
\hline & & & p.Lys117Asn & c. $351 \mathrm{G}>\mathrm{T}$ \\
\hline & & \multirow{2}{*}{146} & p.Ala146Thr & c. $436 \mathrm{G}>\mathrm{A}$ \\
\hline & & & p.Ala146Val & c. $437 C>T$ \\
\hline$B R A F$ & 15 & 600 & p.Val600Glu & c.1799T > A \\
\hline
\end{tabular}


Table 3. Selected techniques used for KRAS, NRAS, and BRAF genotyping [13, 34-39]

\begin{tabular}{|c|c|c|c|}
\hline Technique & $\begin{array}{l}\text { Sensitivity of } \\
\text { the method* }\end{array}$ & $\begin{array}{l}\text { Time of } \\
\text { analysis }\end{array}$ & Details \\
\hline $\begin{array}{l}\text { Sanger sequencing } \\
\text { and } \\
\text { pyrosequencing }\end{array}$ & $10-20 \%$ & $\begin{array}{l}5-10 \text { working } \\
\text { days }\end{array}$ & $\begin{array}{l}\text { Quite low sensitivity, the result requires interpretation by an experienced } \\
\text { user, relatively low cost, the ability to detect new mutations, usually do } \\
\text { not have CE IVD. This method is selected by approx. } 20 \% \text { of laboratories } \\
\text { performing KRAS/NRAS/BRAF determinations }\end{array}$ \\
\hline $\begin{array}{l}\text { Methods based on } \\
\text { allele-specific PCR - } \\
\text { commercial kits }\end{array}$ & $1-5 \%$ & $\begin{array}{l}\text { Less than } 5 \\
\text { working days }\end{array}$ & $\begin{array}{l}\text { Reliable and repeatable techniques based on PCR, have high sensitivity } \\
\text { and high specificity, the ability to detect specific mutations, require many } \\
\text { molecular probes to be used, have CE IVD. This method is selected by } \\
\text { approx. } 50 \% \text { of laboratories performing KRAS/NRAS/BRAF determinations }\end{array}$ \\
\hline $\begin{array}{l}\text { Next-generation } \\
\text { sequencing (NGS) - } \\
\text { commercial kits }\end{array}$ & $0.1 \%-10 \%$ & $\begin{array}{l}\text { Around } 10 \\
\text { working days } \\
\text { or more }\end{array}$ & $\begin{array}{l}\text { Lack of full validation, expensive, TAT is usually longer due to the need of } \\
\text { samples pooling, require interpretation by an experienced user, usually do } \\
\text { not have CE IVD. This method is selected by approx. } 30 \% \text { of laboratories } \\
\text { performing KRAS/NRAS/BRAF determinations }\end{array}$ \\
\hline
\end{tabular}

*The minimal content of cells with a mutation in relation to normal cells

\section{Selected methods used in molecular diagnostics}

The direct Sanger sequencing method is widely recognised as the "golden standard" in DNA mutation detection. The method can be used to determine all mutations in the tested genes, i.e. KRAS, NRAS, and BRAF, allowing for accurate mutation naming both at nucleotide and amino acid levels.

The advantage of this method is the detection of all possible changes in the analysed region of DNA, with the exception of large rearrangements of whole exon(s).

Specific mutations in KRAS/NRAS/BRAF genes can also be identified using commercial qPCR sets, which contain ready-to-use reagents, positive controls, and endogenous controls enabling PCR inhibitors detection. These kits identify the most frequent mutations in KRAS codons 12 , $13,59,61,117$, and 146, and NRAS codons: $12,13,61,117$, and 146, and BRAF codon 600.

Importantly, the very high sensitivity of this type of method makes them especially prone to cross contamination of specimens. For example, if a microtome is not thoroughly cleaned after cutting a sample with a high mutation load, the next sample to be cut is at risk of being contaminated by remnants of the previous one, which can lead to false-positive results. To avoid this, the other, less sensitive method, i.e. Sanger sequencing, can be used. Nevertheless, when macrodissection is not possible, high-sensitivity tests may be highly beneficial.

The technological solutions for rapid, sensitive, and less-laborious detection of somatic mutations develop constantly. There is already a fully automated, CE IVD certified platform (Idylla, Biocartis) available. This device automatically isolates DNA form paraffin scraps and, subsequently, performs qPCR reactions, all within 4-5 hours. Nonetheless, despite automatisation, pathological examination prior to molecular testing is required, and only samples containing more than $10 \%$ of neoplastic fixation can be proceeded. According to its producer, the limit of detection (LOD) reaches 5\% [40].

At the moment, the choice of diagnostic method depends on individual laboratory preferences. Nevertheless, regardless of the technology, thorough validation of the method it is crucial, which includes:

- definition of minimum amount of material and minimal thickness of paraffin scraps for DNA extraction,

- determination of the type of fixatives that can be used for biological material without the risk of negative impact on molecular procedures,

- definition of the quantity, quality, and concentration of the DNA sample that can be processed,

- determination of threshold value to distinguish the mutant from the normal variant,

- verification of test sensitivity (e.g. using dilution series),

- application of the reference method to verify the obtained results,

- ascertainment of method repeatability.

Furthermore, it is crucial to strictly adhere to the procedures, using positive and negative controls in each run and undergoing internal quality control. In addition, regular participation in external quality controls is equally important. Such international programs are annually carried out by EMQN (European Molecular Genetics Quality Network), UKNEQAS (United Kingdom National External Quality Assessment for Molecular Genetics), Colon EQA (European Society of Pathology in collaboration with the University of Leuven), and others.

According to recommendations, the sensitivity of the method should oscillate around $1-5 \%$ of tumour cells for allele-specific PCR methods and 10-20\% for direct sequencing (Fig. 4). Regarding the specificity, at least seven typical changes in codons 12 and 13, variants in codons 59, 61,117 , and 146 of KRAS and NRAS, and variants occurring in codon 600 of the BRAF gene should be detected.

For the purpose of accreditation, the laboratory should obtain DNA in a minimum of $95 \%$ of cases and demonstrate correctness of analyses at the level of 97\% [31, 36-38]. The acceptable turnaround time of the entire procedure should not exceed a maximum of 10 working days [36].

Although mutations detected using qPCR are automatically named, in the case of Sanger sequencing mutation, the name is usually assigned and verified by a diagnostician. Therefore, to avoid misinterpretation, it is extremely important to refer to a proper reference sequence (e.g. 

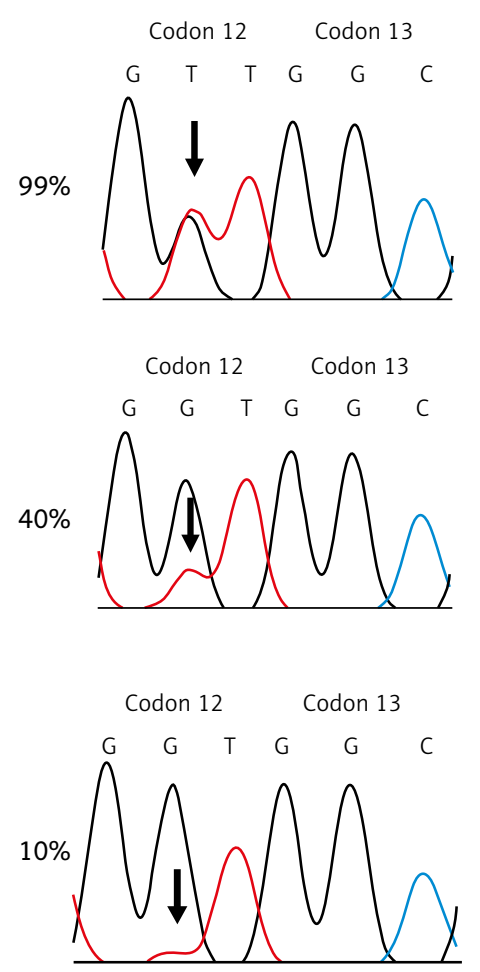
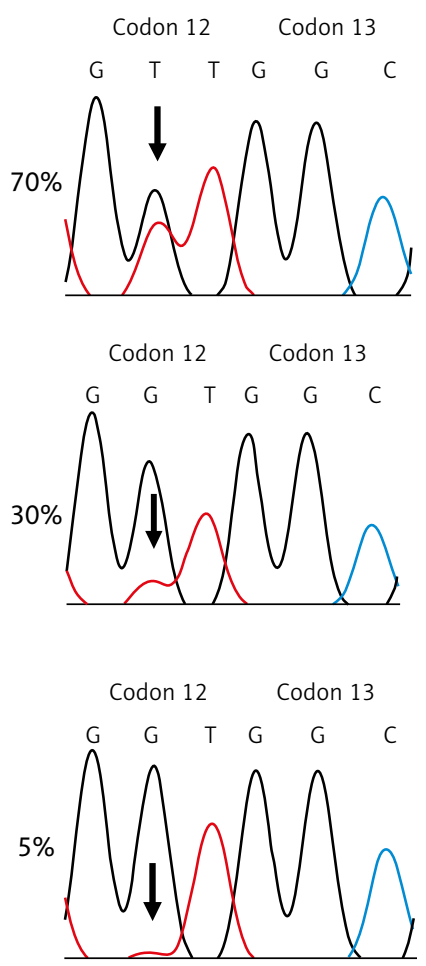
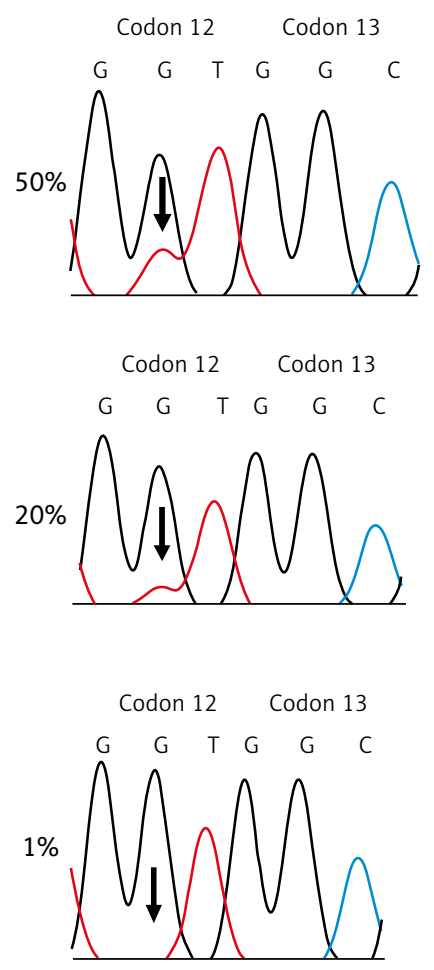

Fig. 4. The figure shows the differences in the ratio of mutant p.Gly12Val allele peak versus wild type, depending on mutation cell count. Fluorograms represent samples with $99 \%, 70 \%, 50 \%, 40 \%, 30 \%, 20 \%, 10 \%, 5 \%$, and 1\% of neoplasm on the background of normal cells. The presence of cells with a mutation can be observed in all dilutions except for the $1 \%$ variant. In the case of the $5 \%$ variant, the signal from the mutant allele is scarcely visible, so the generally accepted sensitivity threshold of direct sequencing is higher and reaches $10 \%$ of cells with the mutation on the wild type cell background

according to LRG: https://www.lrg-sequence.org/) and to use standard mutation nomenclature as defined by HGVS (https://varnomen.hgvs.org/).

Proper and clear reporting of genetic test results is an extremely important issue, which in the case of somatic and tumour mutation diagnostics should be treated as a supplement to histopathological examination. Hence, information from pathological verification, concerning diagnosis and the percentage of neoplastic fixation in a specimen for molecular examination, is obligatory [37]. As stated above, pathological evaluation is crucial with respect to selection of the best methodology, and together with the reason for testing is indispensable for correct interpretation of the results. Consequently, according to European recommendations on mutation identification in KRAS, NRAS, and BRAF genes, the molecular test result should be submitted to the pathologist, who incorporates it into the full characteristics of the paraffin-embedded material or biopsy [31].

\section{Alternative methods of KRAS/NRAS/BRAF testing}

Analysis of circulating tumour DNA (ctDNA) is a relatively novel and valuable diagnostic method. In this strategy, mutation analysis is performed on DNA secreted by tumour cells into the bloodstream. Whenever a cancer sample cannot be obtained, the ctDNA analysis remains the only option. Mutation detection can be performed using ctDNA-dedicated high-sensitivity qPCR tests. Similarly, only dedicated tubes can be used for blood collec- tion. Such tubes contain stabilisers preventing lysis of nucleated blood cells and the release of their genomic DNA, which, if present in the sample, would quantitatively dominate over ctDNA, and finally cause false results. Although ctDNA fraction is generally regarded as a valuable diagnostic material because it represents the entire tumour heterogeneity (not only its fragment, as in the case of standard FFPE tumour specimens testing), it is not recommended that the ctDNA be analysed when a tissue material is available. This is due to the fact that is some studies mutations detected in the tumour samples were not found at the ctDNA level [41].

Large-scale techniques such as next-generation sequencing (NGS) can also be used for genetic diagnostics in targeted therapy qualification analysis. The technology makes it possible to simultaneously analyse coding sequences of many genes, the entire exome, or even the whole-genome. Currently, in CRC only three molecular markers, KRAS, NRAS, and BRAF need to be evaluated by molecular testing; therefore, this laborious and still expensive approach is rarely used. Nevertheless, its sensitivity is very high - even less than 0.1\% DNA with mutation in relation to the wild type DNA can be detected. This makes NGS a very good tool for tissue and ctDNA analysis. In the near future, NGS will probably become more popular because it will be routinely used to assess emerging genetic prognostic biomarker - tumour mutation burden (TMB), which plays a very important role in the immunological therapy of CRC [34]. 


\section{Conclusions}

Routine molecular diagnostics for targeted therapy is a relatively new discipline. Therefore, it is particularly important to develop standards and guidelines for material selection for molecular studies, and for mutation detection itself. In this initial phase, one should focus on a reliable examination of the basic prognostic factors for targeted therapies, which in the case of metastatic colorectal carcinoma include mutations in: KRAS, NRAS, and BRAF genes. The results of the molecular analysis depend on the preservation and fixation of large intestine tumour specimens. The prepared specimen is fixed in $10 \%$ buffered formalin (4\% formaldehyde aqueous solution buffered to $\mathrm{pH}$ 7.2).

At the beginning of 2017, the American Society for Clinical Pathology, the College of American Pathologists, the Association for Molecular Pathology, and the American Society of Clinical Oncology developed guidelines for laboratories performing diagnostic genetic tests aimed at qualification of patients for targeted therapies [31].

According to these recommendations, metastatic or recurrence tissue should be tested preferentially; however, in the absence or insufficient quantity, the primary tumour can also be examined. The reference material for the test is tissue embedded in a paraffin block; however, use of cytological preparations or ctDNA is not excluded. Qualification of the material to molecular analysis must always be carried out by a pathomorphologist and should take into account its quality and quantity as well as the content of neoplastic tissue. Laboratories should have methods capable of detecting at least $5 \%$ of DNA with a mutation in relation to the wild type DNA, they take into account the sensitivity of the method (LOD - limit of detection), and, when necessary, to perform micro/macrodissection in order to select the cancer cell population. The content of neoplastic tissue should be at least twice as high as the sensitivity of the method used. Extended RAS panel including the KRAS and NRAS genes (evaluation of mutations in codons 12, 13, $59,61,117$, and 146 ) should be routinely performed; also analysis of codon 600 of the BRAF gene, requires consideration in each case. The result should be obtained in the shortest possible time, which should not exceed 10 working days. The report on the diagnostic genetic test should include the result of the study, a clearly defined interpretation understandable for the clinical oncologist and pathomorphologist, as well as the description and scope of the applied methodology. Laboratories should have validated the entire diagnostic process from material collection to the final report of the result. Internal quality controls and active participation in external quality assessment are highly recommended.

However, it should be remembered that even in the group of patients in whom the KRAS, NRAS, and BRAF mutation have not been detected, not all individuals will benefit from targeted therapy. This may be due to mutations in other genes encoding EGFR receptor-mediated signalling pathways such as PIK3CA or PTEN, which could limit the effectiveness of targeted treatment. Therefore, it is necessary to constantly search for new therapeutic goals, and thus to implement the widest possible panel of modern methods of molecular biology.

The authors declare no conflict of interest.

\section{References}

1. Didkowska J, Wojciechowska U. Cancer in Poland in 2013. Krajowy Rejestr Nowotworów. Centrum Onkologii Instytut im. M. Skłodowskiej-Curie. Warszawa 2015: 48-49.

2. Cunningham D, Humblet $Y$, Siena S, et al. Cetuximab monotherapy and cetuximab plus irinotecan in irinotecan-refractory metastatic colorectal cancer. N Engl J Med 2004; 351: 337-345.

3. Gross ME, Zorbas MA, Danels YJ, et al. Cellular growth response to epidermal growth factor in colon carcinoma cells with an amplified epidermal growth factor receptor derived from a familial adenomatous polyposis patient. Cancer Res 1991; 51: 1452-1459.

4. Radinsky R, Risin S, Fan D, Dong Z, Bielenberg D, Bucana CD, Fidler IJ. Level and function of epidermal growth factor receptor predict the metastatic potential of human colon carcinoma cells. Clin Cancer Res 1995; 1: 19-31.

5. Messa C, Russo F, Caruso MG, Di Leo A. EGF, TGF-alpha, and EGF-R in human colorectal adenocarcinoma. Acta Oncol 1998; 37: 285289.

6. Kluftinger AM1, Robinson BW, Quenville NF, Finley RJ, Davis NL. Correlation of epidermal growth factor receptor and c-erbB2 oncogene product to known prognostic indicators of colorectal cancer. Surg Oncol 1992; 1: 97-105.

7. Wojtukiewicz MZ, Sierko E. Leczenie celowane u chorych na raka jelita grubego. Onkol Prakt Klin 2007; 3: 286-297.

8. Cree IA, Deans S, Ligtenberg MJL, et al. Guidance for laboratories performing molecular pathology for cancer patients. I Clin Pathol 2014; 67: 923-931.

9. Sluijter CE, van Lonkhuijzen LRCW, Slooten H-J, Nagtegaal ID, Overbeek LIH. The effects of implementing synoptic pathology reporting in cancer diagnosis: a systematic review. Virchows Arch 2016; 468: 639-649.

10. Jessup JM, Goldberg RM, Asare EA, et al. Colon and Rectum. American Joint Committee on Cancer 2017. MB Amin, et al. (Eds.), AJCC Cancer Staging Manual. 8th ed. Springer, New York: 251-274.

11. Brierley JD, Gospodarowicz MK, Wittekind C, et al. (Eds.). TNM Classification of Malignant Tumours. 8th ed. Wiley Blackwell, Oxford 2017.

12. Amin MB, Greene FL, Edge SB, et al. The Eighth Edition AJCC Cancer Staging Manual: Continuing to Build a Bridge From a Population-Based to a More "Personalized" Approach to Cancer Staging. Ca Cancer J Clin 2017; 67: 93-99.

13. Tysarowski A, Fabisiewicz A, Kolasa I, et al. Walidacja wybranych technik molekularnych oznaczania mutacji w kodonie 12 i 13 genu K-RAS przeprowadzona w pięciu ośrodkach badawczo-naukowych Polski. Onkol Prakt Klin 2008; 4: 232-244.

14. Schubbert S, Shannon K, Bollag G. Hyperactive Ras in developmental disorders and cancer. Nat Rev Cancer 2007; 7: 295-308.

15. Konstantinopoulos PA, Karamouzis MV, Papavassiliou AG. Post-translational modifications and regulation of the RAS superfamily of GTPases as anticancer target. Nat Rev Drug Discov 2007; 6: 541-555.

16. Bourne HR, Sanders DA, McCormick F. The GTPase superfamily: conserved structure and molecular mechanism. Nature 1991; 349: 117-127.

17. Downward J. Ras signalling and apoptosis. Curr Opin Genet Dev 1998; 8: 49-54.

18. Crespo P, León J. Ras proteins in the control of the cell cycle and cell differentiation. Cell Mol Life Sci 2000; 57: 1613-1636.

19. Macaluso M, Russo G, Cinti C, Bazan V, Gebbia N, Russo A. Ras family genes: an interesting link between cell cycle and cancer. J Cell Physiol 2002; 192: 125-130.

20. Ellis CA, Clark G. The importance of being K-Ras. Cell Signal 2000; 12: $425-434$ 
21. Capella G, Cronauer-Mitra S, Peinado MA, Perucho M. Frequency and spectrum of mutations at codons 12 and 13 of the c-K-ras gene in human tumors. Environ Health Perspect 1991; 93: 125-131.

22. Bos JL. Ras oncogenes in human cancer: a review. Cancer Res 1989; 49: 4682-4689.

23. Bazan V, Migliavacca M, Zanna I, et al. A Specific codon 13 K-RAS mutations are predictive of clinical outcome in colorectal cancer patients, whereas codon $12 \mathrm{~K}$-RAS mutations are associated with mucinous histotype. Ann Oncol 2002; 13: 1438-1446.

24. Senagore AJ, Biener JT. A newly identified pattern of K-RAS mutations at codons 12 and 13 is associated with long-term survival in colorectal cancer. Surgery 1997; 122: 765-770.

25. Bartczak M, Sałagacka A, Mirowski M, Balcerczak E. Status genu K-RAS jako czynnik prognostyczny i predykcyjny w raku jelita grubego. Nowotwory. Journal of Oncology 2010; 60: 147-156.

26. Wang Y, You M, Wang Y. Alternative splicing of the K-ras gene in Mouse tissues and cell lines. Exp Lung Res 2001; 27: 255-267.

27. Plowman SJ, Berry RL, Bader SA, Luo F, Arends MJ, Harrison DJ, Hooper ML, Patek CE. K-ras $4 \mathrm{~A}$ and $4 \mathrm{~B}$ are co-expressed widely in human tissues, and their ratio is altered in sporadic colorectal cancer. J Exp Clin Cancer Res 2006; 25: 259-267.

28. Garnett MJ, Marais R. Guilty as charged: B-RAF is a human oncogene. Cancer Cell 2004; 6: 313-319.

29. Davies H, Bignell GR, Cox C. Mutations of the BRAF gene in human cancer. Nature 2002; 417: 949-954.

30. De Roock W, Claes B, Bernasconi D, et al. Effects of KRAS, BRAF, NRAS, and PIK3CA mutations on the efficacy of cetuximab plus chemotherapy in chemotherapy-refractory metastatic colorectal cancer: a retrospective consortium analysis. Lancet Oncol 2010; 11: 753-762

31. Sepulveda AR, Hamilton SR, Allegra CJ, et al. Molecular Biomarkers for the Evaluation of Colorectal Cancer: Guideline From the Amer ican Society for Clinical Pathology, College of American Pathologists, Association for Molecular Pathology, and American Society of Clinical Oncology. J Mol Diagn 2017; 19: 187-225.

32. http://www.mz.gov.pl/leki/refundacja/lista-lekow-refundowanych-obwieszczenia-ministra-zdrowia/ (accessed: 27.06.2017).

33. National Comprehensive Cancer Network (NCCN). Clinical Practice Guidelines in Oncology (NCCN Guidelines). Colon Cancer. Version 2.2016. Available from: www.nccn.org/patients (accessed: February 2017).

34. Chen G, Yang Z, Eshleman JR, Netto GJ, Lin M-T. Diagnostics for Precision Medicine in Colorectal Cancer: Current Status and Future Perspective. Biomed Res Int 2016; 2016: 9850690.

35. General report, ESP Colon EQA 2017, European Society of Pathology, date of report: 04.10.2017.

36. Linardou H, Briasoulis E, Dahabreh IJ, et al. All about KRAS for clinical oncology practice: gene profile, clinical implications and laboratory recommendations for somatic mutational testing in colorectal cancer. Cancer Treat Rev 2011; 37: 221-233.

37. van Krieken JH, Jung A, Kirchner T, et al. KRAS mutation testing for predicting response to anti-EGFR therapy for colorectal carcinoma: proposal for an European quality assurance program. Virchows Arch 2008; 453: 417-431.

38. Gulley ML, Braziel RM, Halling KC, et al. Molecular Pathology Resource Committee, College of American Pathologists. Clinical lab oratory reports in molecular pathology. Arch Pathol Lab Med 2007 131: 852-863.

39. Shackelford RE, Whitling NA, McNab P, Japa S, Coppola D. KRAS Testing: A Tool for the Implementation of Personalized Medicine. Genes Cancer 2012; 3: 459-466.

40. Biocartis, Idylla specification, www.biocartis.com.

41. Vidal J, Muinelo L, Dalmases A, et al. Plasma ctDNA RAS mutation analysis for the diagnosis and treatment monitoring of metastatic colorectal cancer patients. Ann Oncol 2017; 28: 1325-1332.

\section{Address for correspondence}

\section{Andrzej Tysarowski}

Department of Pathology and Laboratory Medicine Department of Molecular and Translational Oncology Maria Skłodowska-Curie Memorial Cancer Centre and Institute of Oncology

5 Roentgena W.K. St.

02-781 Warsaw, Poland

e-mail: a.tysarowski@coi.waw.pl

Submitted: 30.04 .2018

Accepted: 27.06 .2018 